\title{
Starburst and old population in $z=3.8$ radio galaxies with Pégase.3
}

\author{
Brigitte Rocca-Volmerange $^{1}$ and Guillaume Drouart ${ }^{2,1}$ \\ ${ }^{1}$ Institut d' Astrophysique de Paris, UPMC/CNRS, 98bis Bd Arago, F-75014 Paris, France \\ email: rocca@iap.fr \\ ${ }^{2}$ European Southern Observatory,Karl Schwarzschild Strasse, 85748 Garching bei München, \\ Germany
}

\begin{abstract}
Distant radio galaxies, hosted by massive ellipticals, follow the galaxy evolution process on an extremely large $(0 \geqslant z \geqslant 7)$ time-scale $\geqslant 10^{12} \mathrm{Gyrs}$, up to primeval galaxies. The new evolutionary code Pégase. 3 predicts on similar time-scales, the coupled stellar and dust emissions of various galaxy types: starbursts and Hubble sequence types. All $\mathrm{z}=0$ templates are fitted on local observations at ages $\simeq 13$ Gyrs (except irregulars at 9 Gyrs). The multi- $\lambda$ spectral energy distributions (SEDs) of two $z=3.8$ radiogalaxies, including the most recent Herschel data from the HeRGÉ consortium, are interpreted in the observer's frame by Rocca-Volmerange et al. (2012) with Pégase.3. The apparent SEDs are fitted at best with the sum of a young starburst and an older early-type population, an AGN simple model is taken into account. These results favor massive gas-rich mergers at work in evolved galaxies at $z \simeq 4$. Massive starbursts would be at the origin of galaxy evolution initiated at the earliest epochs $\left(z_{f o r} \geqslant 10\right)$. The possible relation with super massive black holes is still debated.
\end{abstract}

Keywords. galaxies: formation, galaxies: evolution, radio continuum: galaxies, infrared: galaxies, stars: formation

\section{Introduction}

High-redshift radio galaxies are known as the most distant stellar populations hosting super massive black holes. From their near-IR morphologies, they are identified with massive early-type galaxies (van Breugel et al. 1998; Lacy et al. 2000; Pentericci et al. 2001). Data interpretation with the evolutionary code Pégase.2 (www2.iap.fr/pegase) of the Hubble $K$-band diagram (De Breuck et al. 2002) shows that the brightest luminosity limit corresponds to powerful radio galaxies hosted by massive elliptical galaxies with baryonic masses $M_{\text {bar,max }} \simeq 10^{12} M_{\odot}$ (Rocca-Volmerange et al. 2004). Populations of radio galaxies were likely discovered in the near-infared with IRAS, ISO and Spitzer: the excesses simultaneously observed in faint galaxy counts at $12 \mu \mathrm{m}, 15 \mu \mathrm{m}$ with ISO (Seymour et al. 2007 and references therein) and at $24 \mu \mathrm{m}$ with Spitzer (Papovich et al. 2004) are interpreted as $9 \%$ of ultra-bright dusty galaxies evolving as ellipticals, likely AGN hosts (Rocca-Volmerange et al. 2007). More recently, distant radio galaxies were observed with Herschel/PACS and SPIRE instruments by the HeRGÉ (Herschel Radio Galaxy Evolution) Project (Seymour et al. 2012). Together with submmillimeter observations, the continuous SEDs of two $z=3.8$ radio galaxies 4C 41.17 and TN J2007-1316, selected for their faint AGN contribution and strong star formation signatures from the HeRGÉ sample, are interpreted with the evolutionary code Pégase.3. Section 1 is a presentation of the template models of early types, Section 2 gives the spectral synthesis results of the $z=3.8$ radio galaxy $4 \mathrm{C} 41.17$ in the observer's frame. Section 3 proposes some preliminary conclusions. 
Table 1. The main free parameters of star formation scenarios (hereafter for elliptical and spiral Sa) in the Pégase. 3 model. The adopted star formation laws are proportional to the current (neutral and molecular) gas mass $M_{\text {gas }}$ with an accretion rate depending on type. The infall time-scale and the age of galactic winds if any, are given. Metal-enrichment and dust amount are computed at all ages from yields and the adopted IMF in the chemical evolution formalism. A variety of IMFs are proposed.

\begin{tabular}{|l|c|c|c|}
\hline Scenario & Star formation rate & Infall time-scale & Galactic wind age \\
\hline Elliptical & $3.3310^{-3} M_{\text {gas }} M_{y r^{-1}}$ & $300 \mathrm{Myrs}$ & $1 \mathrm{Gyr}$ \\
\hline Spiral Sa & $0.7110^{-3} M_{\text {gas }} M_{y r^{-1}}$ & $2800 \mathrm{Myrs}$ & no winds \\
\hline
\end{tabular}

\section{Template models of early-type galaxies}

Following the readme of the code Pégase.2 (freely accessible at www2.iap.fr/pegase), input parameters for an elliptical and a spiral galaxy are presented in Table 1. Figure 1 illustrates the time evolution of various (total galaxy, stellar and gas) masses for the elliptical galaxy. The parameter set of Table 1 defines the star formation time-scale varying from 1 Gyr to 10 Gyrs from ellipticals to spirals (see also fig.3 of RoccaVolmerange et al. 2004). Synthetic libraries of SEDs evolving from ages of 0 to $20 \mathrm{Gyr}$ are built to simulate the evolution of instantaneous starbursts and of a variety of galaxy types: elliptical (E), lenticular (S0), spiral (Sa, Sb, Sbc, Sc, Sd) and Magellanic irregular (Im) galaxies. Scenarios are robust at $z=0$, all are fitting local observed colours by types. The star formation process is initiated at the so-called redshift of formation $z_{\text {for }}=10$, implying the age of 13 Gyrs (only 9 Gyrs for Irregulars) for local $z=0$ templates. Changing $z_{f o r}$ to 20 or 30 will only vary ages of local templates by $\leqslant 0.4$ Gyrs according to the $z$-cosmic time relation, implying small variations in the SED templates. We adopt the following parameters : $\mathrm{H}_{0}=70 \mathrm{~km} . \mathrm{s}^{-1} M p c^{-1}, \Omega_{M}=0.3, \Omega_{\Lambda}=0.7$. For all scenarios, the

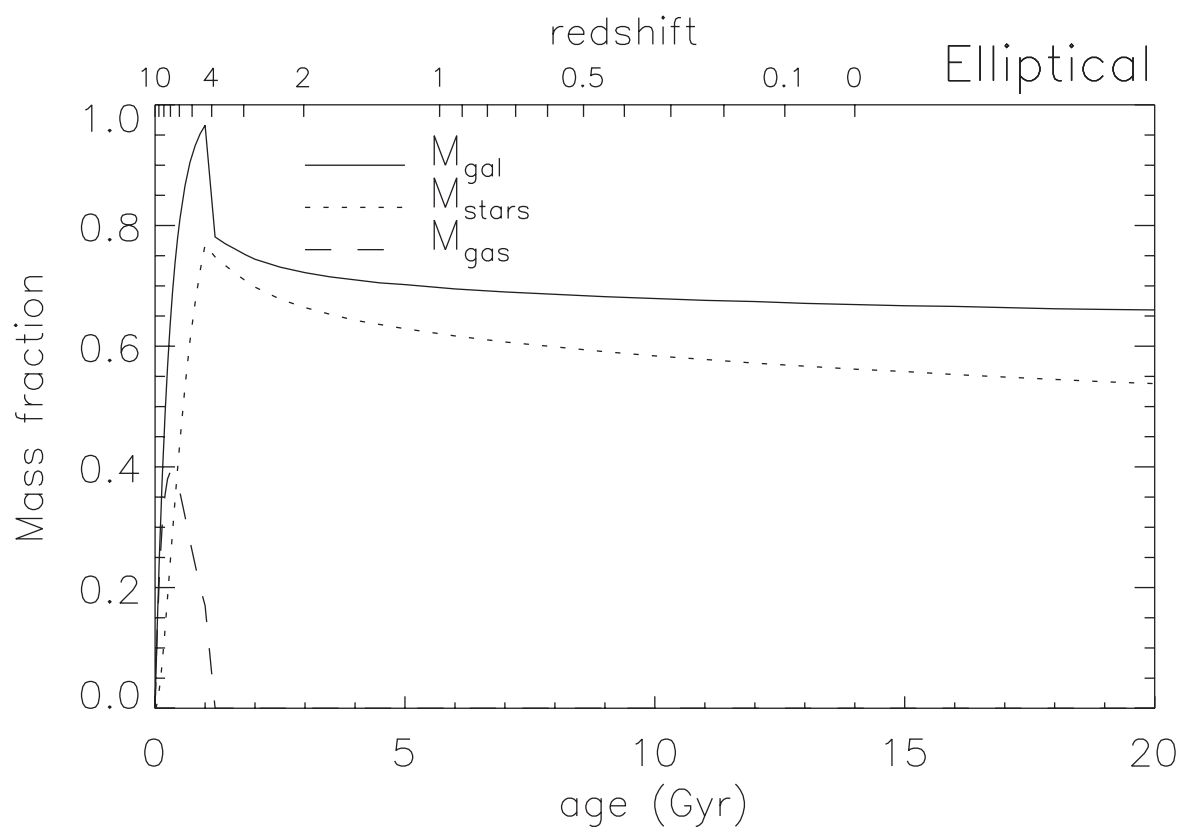

Figure 1. Mass evolution predicted for the elliptical galaxy. 
initial mass function (IMF) is standard (Kroupa et al. 1993): changing IMF does not significantly modify the final results. After checking that one single component is clearly insufficient, an automatic procedure based on the $\chi^{2}$ minimum algorithm finds the best fit to the observations with the sum of two templates of the synthetic libraries (starburst + Hubble type galaxies).

\section{Multiple components of distant Radio Galaxies}

The new version of the code Pégase.3 (Fioc et al. 2012, in preparation) predicts, in a coherent way, the evolution of the stellar emission, the metal and dust content, the attenuation and the reemission by dust. With this new version, synthetic spectral energy distributions (SEDs) are continuously created over a large wavelength coverage from the far-UV to the submillimeter at all ages. Local templates are converted to the high- $z$ observer's frame after corrections of distance, universe expansion and evolution by type ( $\mathrm{k}+\mathrm{e}$ corrections) using the cosmic time- $z$ relation (Rocca-Volmerange \& Guiderdoni 1988). Both far-IR and optical emissions of the $z=3.8$ radio galaxy $4 \mathrm{C} 41.17$ are remarquably well fitted by a massive starburst in an evolved phase (so-called post-burst). It is worth to recall the energy distributions in the two domains are tightly correlated through the total Lyman continuum photon number $N_{L y c}$ emitted by young and massive stars whose number scales with the star formation rate: The absorbed fraction is dustscattered and re-emitted by cold grains in the far-IR while the not-absorbed fraction is traced by photospheric emission in the optical. Moreover an evolved population at age

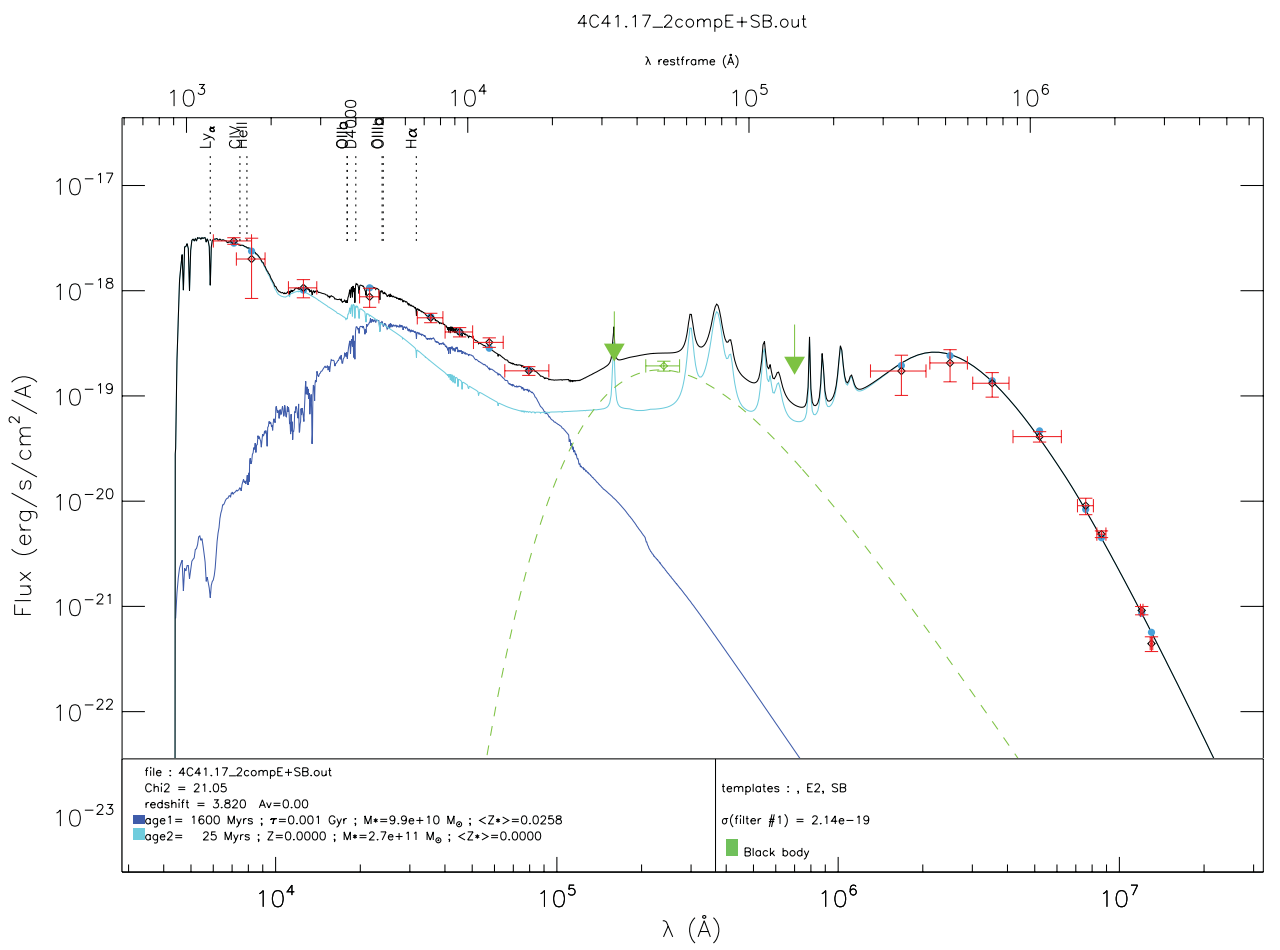

Figure 2. The three components of the $z=3.8$ radio galaxy $4 \mathrm{C} 41.17$ SED modeled with Pégase. 3 within error bars: a young (25 Myrs) starburst, the $z=3.81 \mu \mathrm{m}$ population (1600 Myrs) and the AGN black-body emission. Details and error bars are discussed in Rocca-Volmerange et al. (2012). 
of 1600 Myrs, typically the $1 \mu \mathrm{m}$ peak of giant stars in early type galaxies, is required to fit the K-band and Spitzer data. Masses are huge and vary within the range $10^{10-12} M_{\odot}$ for starbursts and evolved galaxies. Mass ratios from 1:1 to 1:10 identify major gas-rich mergers as well as the possibility of jet-cloud interactions. In most cases, interacting galaxies are no-primitive clouds. In a near future, a better statistics on ages and masses as well as on the interpretation of the AGN component (Drouart et al. 2012) will be derived from the complete analysis of the HeRGÉ sample.

\section{Conclusions}

We show (Rocca-Volmerange et al. 2012, submitted) that the best-fitting SED of the $z=3.8$ radio galaxy $4 \mathrm{C} 41.17$ is the sum of two stellar components: an evolved starburst, observed at the age of $25 \mathrm{Myr}$, dominant in the far-IR Herschel/submm and in the optical plus an evolved ( $\geqslant 1$ Gyr at $z=4$ ) population peaking at $1 \mu \mathrm{m}$ (rest frame) in the Spitzer domain. This older component was already found from the $K-z$ Hubble diagram (RoccaVolmerange et al. 2004). Masses of all components are confirmed to be huge $\left(10^{10-12} M_{\odot}\right)$. The possibility of a rapid evolutionary process at early epochs is favored. The link to the black hole growth is still debated.

\section{References}

De Breuck, C., van Breugel, W., Stanford, S. A., et al. 2002, AJ, 123, 637

Drouart, G., De Breuck, C., Vernet, J., Laing, R. A., Seymour, N. et al. 2012, A\&A, 548, 45

Fioc, M. \& Rocca-Volmerange, B. 1997, A\&SA, 326, 950

Fioc, M., Rocca-Volmerange, B. \& Dwek, E. 2012, in preparation

Lacy, M., Bunker, A. J., \& Ridgway, S. E. 2000, ApJ (Letters), 120, L68

Papovich, C., Dole, H., Egami, E., Le Floc'h, E., Pérez-Gonzàlez, P. G., et al. 2004, ApJS, 154, 70

Pentericci, L., McCarthy, P., Röttgering, H., et al. 2001, ApJS, 135, 63

Rocca-Volmerange, B. \& Guiderdoni, B. 1988, A\&AS, 75, 93

Rocca-Volmerange, B., Le Borgne, D., De Breuck, C., Fioc, M., \& Moy, E. 2004, A\&A, 415, 931

Rocca-Volmerange, B., de Lapparent, V., Seymour, N., \& Fioc, M. 2007, A\&A, 475, 801

Seymour, N., Rocca-Volmerange, B., \& de Lapparent, V. 2007, A\&A, 475, 791

Seymour, N., Altieri, B., De Breuck, C., et al. 2012, ApJ, 755, 146

van Breugel, W., Stanford, S., Spinrad, H., et al. 1998, ApJ, 502, 614 